\title{
Erratum to: Dendrochemical investigation \\ on hexachlorocyclohexane isomers (HCHs) in poplars \\ by an integrated study of micro-Fourier transform infrared spectroscopy and gas chromatography
}

\author{
Roberta Bernini $^{1}$ - Claudia Pelosi ${ }^{2}$ Isabella Carastro ${ }^{1} \cdot$ Rachele Venanzi $^{1}$. \\ Alfredo Di Filippo ${ }^{1} \cdot$ Gianluca Piovesan $^{1} \cdot$ Bruno Ronchi $^{1} \cdot$ Pier Paolo Danieli $^{1}$
}

Published online: 28 March 2016

(c) Springer-Verlag Berlin Heidelberg 2016

\section{Erratum to: Trees}

\section{DOI 10.1007/s00468-015-1343-8}

Unfortunately, the column head of Table 2 of the original article was wrongly published online. The corrected Table 2 is given in the following page.
The online version of the original article can be found under doi:10.1007/s00468-015-1343-8.

Pier Paolo Danieli

danieli@unitus.it

Dipartimento di Scienze Agrarie e Forestali (DAFNE), Università degli Studi della Tuscia, Via San Camillo de Lellis, 01100 Viterbo, Italy

2 Dipartimento di Scienze dei Beni Culturali (DISBEC), Università degli Studi della Tuscia, Largo dell'Università, 01100 Viterbo, Italy
Table 2 Presence/absence of micro-FTIR peaks of $\mathrm{HCHs}$ in treerings (1991-2012) and estimated abundance

\begin{tabular}{|c|c|c|c|c|c|c|}
\hline \multirow[t]{3}{*}{ Year } & \multirow{3}{*}{$\begin{array}{l}\text { Unpolluted area } \\
\text { Control }\end{array}$} & \multicolumn{5}{|c|}{$\mathrm{HCHs}$ polluted area } \\
\hline & & \multirow{2}{*}{$\begin{array}{l}\text { Far from } \\
\text { river bank } \\
1^{*}\end{array}$} & \multicolumn{4}{|c|}{ Close to river bank } \\
\hline & & & $2 *$ & 3 & 4 & 5 \\
\hline 2012 & 0 & ++ & ++ & + & ++ & + \\
\hline 2011 & 0 & & + & + & + & + \\
\hline 2010 & 0 & ++ & 0 & ++ & ++ & 0 \\
\hline 2009 & 0 & ++ & 0 & + & 0 & 0 \\
\hline 2008 & 0 & ++ & + & ++ & 0 & 0 \\
\hline 2007 & 0 & + & ++ & ++ & 0 & 0 \\
\hline 2006 & 0 & ++ & ++ & + & + & 0 \\
\hline 2005 & 0 & + & ++ & ++ & ++ & ++ \\
\hline 2004 & 0 & ++ & ++ & ++ & ++ & 0 \\
\hline 2003 & 0 & 0 & 0 & + & ++ & 0 \\
\hline 2002 & 0 & ++ & 0 & ++ & ++ & 0 \\
\hline 2001 & 0 & 0 & 0 & + & ++ & + \\
\hline 2000 & 0 & 0 & ++ & & + & + \\
\hline 1999 & 0 & ++ & ++ & & ++ & ++ \\
\hline 1998 & 0 & ++ & ++ & ++ & ++ & ++ \\
\hline 1997 & 0 & ++ & ++ & + & ++ & ++ \\
\hline 1996 & 0 & 0 & ++ & + & ++ & + \\
\hline 1995 & 0 & + & & & ++ & + \\
\hline 1994 & 0 & + & & ++ & + & 0 \\
\hline 1993 & 0 & 0 & & ++ & ++ & + \\
\hline 1992 & 0 & 0 & & ++ & 0 & + \\
\hline 1991 & 0 & & & ++ & + & + \\
\hline
\end{tabular}

0 undetected, ++ detected, high level, + detected, moderate, blank measure not possible due to wood rot or irregular sample surface

* Trees analyzed in the GC-ECD 